\title{
I programmi per la formazione degli insegnanti di italiano all'estero*
}

\section{Francesco Sabatini}

\section{Questioni generali}

Nella prima parte di questa relazione si fa brevemente il punto su alcuni presupposti generali, acquisiti mediante i dibattiti con i colleghi canadesi, ai quali va il più vivo ringraziamento per la ricchezza e chiarezza delle informazioni che hanno fornito.

Nelle due parti successive si prospettano indicazioni e suggerimenti che, lungi dall'esprimere ingenerose critiche alle istituzioni nelle quli si formano gli insegnanti di italiano all'estero, rappresentano il risultato a cui si è pervenuti concordemente attraverso quei dibattiti.

1.1. Necessità di iniziative specifiche e di un quadro di vera politica culturale.

Se nuove iniziative partono oggi dal nostro paese per promuovere la diffusione della lingua e della cultura italiana all'estero ciò si deve alla concomitanza di due fattori. Da una parte, si hanno i primi segni che si sta formando anche da noi una "diplomazia con una vocazione culturale." 1 D'altra parte, tra gli studiosi ed esperti di insegnamento delle lingue e di trasmissione della cultura comincia a diffondersi una competenza più specifica e una maggiore sensibilità per i complessi problemi di quella che troppo genericamente e unilateralmente si chiama "diffusione della lingua e della cultura." Non ho titoli per soffermarmi sul primo fenomeno, ma avverto che già in una circostanza come questo incontro in Canada, le ipotesi di studio e di lavoro acquistano un significato più preciso perché si inquadrano in una cornice di politica culturale di parte italiana.

Alle origini di questo Convegno c'è infatti l'intenzione, da parte di diplomatici ed esperti, di avviare più precisi rilevamenti delle situazioni in cui si deve operare e di stabilire una vera collaborazione con gli esponenti degli studi di italiano all'estero e con le istituzioni ufficiali interessate. Specialmente in presenza dei vasti fenomeni di assestamento sociale creati dall'emigrazione, iniziative efficaci nel campo educativo e culturale non sono immaginabili senza un'attenta programmazione e un'autentica cooperazione tra organismi pienamente responsabili e dotati di capacità di intervento. 
Affrontando il problema della formazione degli insegnanti di italiano all'estero e dell'impostazione dei programmi educativi nei vari livelli scolastici entriamo necessariamente in un quadro, appunto, di intese bilaterali e di impegni ufficiali.

1.2. Conoscere il grado di qualificazione degli insegnanti di italiano2. Rilevamenti compiuti più volte, e in diversi paesi, hanno messo in evidenza un dato che si ripresenta puntualmente anche in Canada e che è da porre al centro di tutti i nostri discorsi: una parte notevole degli insegnanti di italiano all'estero ha una scarsa qualificazione professionale. Ė questo un dato molto complesso, che non puo essere interpretato in base a superficiali valutazioni delle capacità e delle attitudini dei singoli. In realtà, il grado di preparazione e, più ancora, di specifica formazione professionale degli insegnanti è innanzi tutto la risultante di due fattori oggettivi: l'intero loro curricolo di studi formativi e la consistenza, vista in prospettiva dagli interessati, delle loro possibilità di impiego professionale.

I problemi di occupazione degli insegnanti di italiano all'estero esulano dalla mia competenza, ma ad essi devo dedicare qui un breve accenno, per la stretta connessione tra questo fattore e lo svolgimento del curricolo di studi universitari.

I relatori delle diverse province canadesi ${ }^{3}$ hanno costantemente messo in evidenza questa situazione: nonstante la forte richiesta generalizzata di insegnamento di italiano nei vari ordini e tipi di scuole, le possibilità di occupare un posto stabile come insegnante di questa lingua sono pochissime. Di conseguenza anche chi (e sono molti) ha interesse e propensione per dedicarsi all'insegnamento dell'italiano preferisce seguire, negli studi universitari, un curricolo principale che di fatto lo qualifica in altre discipline e cioè, di solito, come insegnante di francese o di spagnolo. Anche in seguito, questi laureati esplicano quasi sempre l'attività didattica principale in un'altra disciplina, mentre l'insegnamento dell'italiano rapprensenta per essi un incarico complementare.

L'incertezza dell'occupazione è dunque un fattore di primo ordine (anche se non l'unico) nel determinare la scarsa preparazione e qualificazione di questi insegnanti.

Si deve aggiungere che la stessa causa influisce negativamente anche in altro modo sugli studi universitari degli aspiranti insegnanti. Dato il collegamento precario tra l'Università e i corsi scolastici di italiano, da questi non giungono facilmente agli istituti universitari quegli stimoli e quelle richieste che impongono modifiche e arricchimenti dei programmi di studio. Ma certamente le limitazioni dell'indirizzo attuale di tali programmi dipendono da alcuni presupposti piu generali che vanno ormai, in ogni caso, largamente rivisti come dirò nella seconda parte di questa relazione. 
1.3. Conoscere $i$ destinatari dei corsi scolastici di italiano: il loro patrimonio di partenza, le loro motivazioni, $i$ loro atteggiamenti.

Vero punto di partenza per l'impostazione del curricolo formativo degli insegnanti dev'essere una migliore conoscenza dei destinatari del loro insegnamento. Un dato ovvio, eppure troppo disatteso nella pratica didattica, è che sussistono notevoli differenze, sotto l'aspetto che qui ci interessa, tra gli ambienti con presenza, più o meno forte, di immigrati di origine italiana e gli ambienti privi di tale presenza. Sono differenze che riguardano sia le oggettive condizioni linguistiche di partenza dei discenti, sia la loro motivazione ad apprendere l'italiano.

a) Il rapporto col patrimonio linguistico e culturale di partenza.

Viene segnalato concordemente da tutte le fonti che gli alunni di famiglie oriunde italiane (specialmente se di immigrazione piuttosto recente), pur presentandosi a volte come già "italofoni," in realtà molto spesso sono soltanto "dialettofoni." 4 Questa loro condizione, che spesso dagli insegnanti non viene considerata affatto o viene affrontata in modo improprio, pone invece problemi specifici, sia sul piano strettamente glottodidattico (necessità di un insegnamento contrastivo che utilizzi anche questo stadio linguistico), sia sul piano psicologico (problemi del confronto con gli alunni non oriundi italiani; ${ }^{5}$ frequente demotivazionne dell'alunno, via via che questi avverte una divergenza con le aspettative iniziali di un facile apprendimento dell'italiano). 6

\section{b) Le diverse motivazioni ad apprendere.}

"Oriundi" e "non oriundi" sono anche, ovviamente, portatori di motivazioni ben diverse nella scelta di apprendere l'italiano. Per la verità, la percentuale di non oriundi nei corsi propriamente scolastici di italiano (escludendo sempre le Università) è molto bassa e quindi il discorso su questa componente è limitato: per i pochi ragazzi non oriundi che scelgono di studiare l'italiano sussistono, data l'età, motivi contingenti e di varia natura (rapporti con parenti e vicini, progetti familiari e simili), non riconducibili a una casistica tipo. Sicché l'insegnante dovrà regolarsi, nei loro confronti, caso per caso. Ma ciò non significa che si possa trascurare il problema della motivazione e, più specificamente, dell'atteggiamento $^{7}$ di quei discenti di altro gruppo etnico che, in contesti multietnici, si interessano alla nostra lingua.

Ben diversa è la situazione per gli oriundi, che costituiscono, almeno in paesi come il Canada, gli USA, l'Australia e l'Argentina, la massa degli iscritti ai corsi di italiano. Attraverso la presenza di questi alunni la problematica dell'emigrazione entra pienamente nel campo scolastico e dell'insegnamento delle lingue. Il tema, 
vasto e complesso, richiede molta attenzione da parte dei pianificatori e operatori dell'insegnamento dell'italiano all'estero per le ragioni che qui dobbiamo richiamare, sia pure di sfuggita. ${ }^{8}$

Il netto aumento globale di richiesta dell'italiano all'estero ha come prima componente proprio il forte risveglio di interesse, nei discendenti dei nostri emigrati, per la lingua dell'antica terra d'origine. Limitarsi a questa constatazione non permette, però, di comprendere il fenomeno e porta, alla fine, a dibattere un falso problema: se davvero la nostra lingua conosca questa stagione favorevole per effetto dell'emigrazione e non più per merito delle sue antiche tradizioni di cultura. Il dilemma "lingua di cultura" o "lingua veicolare e di comunicazione sociale" presenta in termini ristretti e impropri una realtà che è molto più varia. Nei paesi di maggiore e stabile afflusso dei nostri emigrati il fattore veramente nuovo e incisivo è, senza dubbio, la pressione delle seconde e terze generazioni degli emigrati. Ma questa richiesta che viene, per cosí dire, "dal basso," in quanto scaturisce da una vicenda sociale come l'emigrazione, non è in contrasto con la tradizionale considerazione dell'italiano come "lingua di cultura." I discendenti degli emigrati chiedono, è vero, di imparare l'italiano per una serie di ragioni particolari e a volte contingenti: perché sono spinti dall'ambiente familiare; perché ritengono di essere facilitati in questo studio da qualche (presunta) conoscenza preesitente della lingua; perché seguono la tendenza generale di acquistare quella specifica "identità culturale" che oggi è molto ricercata nei paesi di forte emigrazione. ${ }^{9}$ Ma è altrettanto certo che questi soggetti aspirano cosi tenacemente al possesso dell'italiano proprio perché si tratta di una riconosciuta lingua di cultura. Il ruolo di "lingua di cultura" è dunque ancora un titolo decisivo per la presenza dell'italiano nel mondo; ma è altreltanto vero che questa funzione oggi viene riaffermata e potenziata soprattutto dalla richiesta di apprendimento che sale dalle comunità dei nostri emigrati.

Più in generale si deve aggiungere, poi, che la "cultura" esportata dal nostro paese si compone di molti altri elementi (oltre a quelli specificamente intellettuali), quali l'artigianato, la moda, la gastronomia, la tecnologia, e che la diffusione della cultura avviene anche attraverso nuovi agenti di mediazione come il cinema, la televisione e l'imponente movimento turistico. In breve, è il potenziale produttivo complessivo di un paese con le nostre dimensioni demografiche, la nostra posizione e le nostre tradizioni, che sostiene l'attuale (crescente, ma precaria) "richiesta" di lingua italiana nel mondo. I fatti fin qui evocati hanno dirette connessioni con la impostazione dei corsi scolastici di italiano e, di conseguenza, con la formazione degli insegnanti che dovranno svolgerli. Nelle fasce scola- 
stiche più basse, dove non vigono ancora criteri di utilità professionale o motivazioni intellettuali, gli alunni sono portatori delle più varie esigenze, inclinazioni e aspirazioni, che l'insegnante deve saper coltivare in vista di interessi piu concreti nel domani di quegli alunni. Non potendo contare, nel caso dell'italiano, su una palese funzione per usi pratici immediati, l'insegnante deve sapere far leva su quelle altre motivazioni che possiamo definire di ordine familiare e latamente culturale. Ma la capacità di cogliere e utilizzare spunti connessi con le nostre culture regionali, con le correnti di scambi commerciali, tecnologici e turistici da e verso il nostro paese, con l'immagine dell'Italia epicentro, nel passato e nel presente, della vita del Mediterraneo - per indicare solo alcuni riferimenti più tipici -, questa capacità può derivare all'insegnante soltanto da un curricolo formativo che attinga a un più largo ventaglio di conoscenze (etnologiche, linguistiche, storiche, nel senso più lato e più ricco, socio-economico) e da un'adeguata preparazione in campo psico-pedagogico.

\section{Lineamenti di curricolo per la formazione (ai vari livelli) degli insegnanti.}

La preparazione e qualificazione degli insegnanti di italiano deve realizzarsi (come già accade nei casi migliori) attraverso fasi diverse. Possiamo individuare tre livelli specifici: il livello della formazione di base, il livello della qualificazione professionale e il livello di perfezionamento e aggiornamento. Esporrò sinteticamente alcune proposte che dovrebbero servire a rendere piu adeguati, sotto il profilo dei "contenuti," i programmi relativi ai tre livelli, con particolare riguardo per il primo e per il terzo.

\subsection{La formazione di base.}

In Canada, come negli USA e nella maggior parte dei paesi esteri, la formazione di base degli insegnanti di italiano avviene fondamentalmente nell'ambito di un solo Dipartimento universitario che è, di solito, quello di lingue e letterature. Già questa circostanza crea una spiccata specializzazione di indirizzo, che di norma tiene lontano lo studente dai corsi di storia, arte, etnologia, ecc., che si tengono in altri Dipartimenti. Si nota poi che tra i corsi delle diverse lingue, quelli per l'italiano pongono, accanto all'insegnamento della lingua, una tematica che è più strettamente di carattere letterario. In sintesi si può osservare che:

a) $i$ corsi di lingua appaiono, in genere, articolati a vari livelli e sono largamente fondati sui principi della moderna glottodidattica;

b) $i$ corsi di storia letteraria sono numerosi e diversificati: accanto ai temi tradizionali, come i grandi trecentisti e il Rinascimento, figu- 
rano sempre più spesso gli scrittori politici, la narrativa dell'Otto e del Novecento e gli scrittori di teatro.

Questo corpus di insegnamenti è ormai un po' dappertutto ben consolidato. Si rileva, invece, una mancanza o una scarsa articolazione, con poche eccezioni, 10 di quei corsi che potrebbero far acquisire al futuro insegnante di lingua e cultura italiana una preparazione per cosí dire "a tutto tondo." Mi riferisco a:

c) corsi di linguistica italiana, ossia di materie come Storia della lingua italiana e Dialettologia italiana, e corsi di Linguistica generale con applicazione all'italiano: materie che permettono di conoscere la reale fisionomia sociolinguistica del nostro paese e la dinamica storica del nostro sistema linguistico, sottoposto, nel tempo, a notevoli diversità di funzioni (da quelle di lingua scritto-letteraria di una ristrettissima élite, a quelle di lingua di comunicazione quotidiana e parlata per una comunità di circa 60 milioni di persone); 11

d) corsi di storia politica, sociale e culturale italiana, che presentino la realtà italiana da diversi punti di vista: non solo attraverso la parte piu "ufficiale" della nostra storia culturale ed artistica, ma attraverso le complesse vicende di formazione della nostra comunità politica e di definizione della nostra fisionomia socioculturale, sullo sfondo dei rapporti con l'ambiente mediterraneo ed europeo, delle molteplici culture locali e delle più recenti trasformazioni economiche, demografiche e sociali, a cui si connettono proprio i grandi fenomeni migratori.

Le aree indicate nei punti $c$ e $d$ cominciano ad essere parzialmente coperte con corsi attivati, in date recentissime, presso i Dipartimenti di italiano o in altri Dipartimenti. ${ }^{12}$ Ma finora nei corsi generali di storia, arte, scienze sociali di altri Dipartimenti il riferimento alla realtà italiana è stato vago o comunque sconnesso dagli altri studi di italianistica; né, d'altra parte, nei piani di studio degli studenti si realizza una larga sintesi tra i corsi dei vari Dipartimenti. In questo modo, alcuni settori di conoscenze di interesse spiccatamente italianistico, come la storia linguistica e la dialettologia italiana, sono stati largamente trascurati. Oppure, in altri casi, settori di grande interesse generale, come la civiltà greca e romana, o la storia del cattolicesimo o la storia della scienza, vengono considerati in una prospettiva che trascende proprio la realtà italiana, che pure ne è intimamente parte.

Ho accennato, finora, ai lineamenti soltanto della formazione di base degli insegnanti. Ma vorrei sottolineare che proprio a questo iivello si puó acquisire decisivamente la capacità di osservare la realtà italiana secondo prospettive più ampie. 


\subsubsection{Il contatto con la situazione locale.}

$E^{\prime}$ utile, a questo punto, scendere in dettagli su un problema essenziale che si presenta nei paesi di forte immigrazione italiana. Per allievi (bambini, ragazzi e anche giovani universitari) che provengono dalle comunità di emigrati o che hanno stretti contatti personali con queste comunità, l'avvicinamento alla lingua e alla cultura italiana non può e non dev'essere un'operazione di "paracadutaggio" nella lontana Italia o solo sul terreno dei grandi temi e valori culturali. Le comunità di oriundi italiani, per quanto siano in corso di diluizione e di smembramento, sono tuttavia ancora internamente legate da molteplici vincoli e vivono in una particolare realtà culturale, nella quale vigono anche peculiari usi linguistici di fondo dialettale-italiano che sono parte integrante della comunicazione effettiva. Questa realtà culturale e linguistica rappresenta una componente della situazione attraverso la quale si deve operare e perciò non se ne può prescindere semplicemente ignorandola o combattendola. Occorre, da parte dell'insegnante, una chiara conoscenza della situazione e una capacità di utilizzare queste che sono pur sempre le "premesse" che predispongono l'allievo a studiare l'italiano.

Sempre per restare vicino alla realtà della grande maggioranza dei suoi allievi, l'insegnante dovrà puntare lo sguardo anche più indietro della loro condizione personale, verso il retroterra delle effettive culture di origine delle loro famiglie e verso la stessa vicenda migratoria: una vicenda cosí profondamente incisa nella personalità di chi l'ha vissuta, deve a un certo punto essere "ritrovata" sull'itinerario di studio, deve cioè essere recuperata e utilizzata.

Sono, tutti questi, "contenuti" essenziali perché l'operazione didattica ( $\mathrm{ma}$ il termine è qui molto povero) risponda alle motivazioni e alle necessità dell'allievo e, aggiungo, dell'insegnante: giacché questi nella quasi totalità dei casi è sullo stesso versante psicologico e socioculturale dei suoi allievi; è cresciuto, figlio di immigrati, come loro.

\subsection{La qualificazione professionale.}

Dopo gli studi universitari, la qualificazione professionale degli insegnanti avviene nell'ambito della Faculty of Education. Ma in poche Università questa facoltà comprende corsi specifici per insegnanti di italiano, sicché gli interessati a questo indirizzo sono, a fortiori, costretti a qualificarsi come insegnanti di altre materie.

Lo sforzo da compiere, dunque, è prima di tutto e principalmente quello di far istituire i corsi di professionalizzazione per insegnanti 
di italiano. Anche in questa sede bisognerà, poi, mantenere quella apertura di orizzonti disciplinari che eviti la polarizzazione sui metodi glottodidattici astrattamente intesi e, riguardo ai "contenuti," sulla tematica letterarica canonica o poco più. Chi si prepara a diventare insegnante di lingua (prima o seconda), oltre alla più scientifica conoscenza e alla migliore padronanza della lingua stessa, dovrà avere una più affinata coscienza degli stretti, inscindibili rapporti della lingua sia con un intero sistema culturale, sia con la personalità del discente e con le finalità che egli persegue, nella sua particolare condizione e situazione.

Queste considerazioni, applicate al settore italianistico, dovrebbero spingere a concentrare l'attenzione sul reale significato che assume, in un dato ambiente e per specifiche aree sociali, la conoscenza della lingua e della cultura italiana. Perció, oltre alle discipline psico-pedagogiche istituzionali e allo sviluppo della competenza linguistica, sembrerebbero particolarmente adatti, sul versante per cosí dire "interno," studi sulle condizioni sociali e sulla fisionomia culturale della comunità di origine italiana e sui rapporti con le altre comunità, e, sul versante "esterno," studi sulle manifestazioni culturali della società italiana che hanno più larga e attuale circolazione nel mondo (cinema, teatro, narrativa; valori storici e ambientali che sono di supporto al turismo; particolari tradizioni artigianali che alimentano gli scambi commerciali) e tutto cio che caratterizza la vita italiana agli occhi degli stranieri. In altri termini, sembra quanto mai opportuno che, nella fase in cui si definisce la preparazione professionale dell'insegnante, siano per lui oggetto di particolare attenzione da una parte la figura del "destinatario" del suo futuro insegnamento e dall'altra gli aspetti e problemi della vita italiana di oggi che costituiscono più facilmente legami con la vita degli altri paesi.

\subsection{Il perfezionamento e l'aggiornamento.}

Per il perfezionamento e l'aggiornamento degli insegnanti esteri di italiano si possono e si debbono prevedere vari tipi di iniziative che non posso qui descrivere singolarmente. In proposito, $\mathrm{mi}$ limito a indicare alcuni criteri generali che riguardano almeno i "corsi di aggiornamento" organizzati, con la collaborazione di parte italiana, in Italia o direttamente nei paesi esteri.

Da vari dibattiti recenti è emersa innanzi tutto l'esigenza che i corsi di aggiornamento non siano concepiti come pure "forniture di dottrina" proposte unilateralmente da parte italiana. Sia i corsi tenuti all'estero, con partecipazione di esperti italiani, sia, e a maggior ragione, quelli tenuti in Italia a gruppi di docenti durante soggiorni solitamente di 4-6 settimane, non possono fondarsi su 
tradizionali lezioni-conferenze dedicate ai canonici temi letterari e di cultura generale, o su generici ripassi di grammatica e conversazione. Il perfezionamento e l'aggiornamento dovranno invece rispondere ad alcuni requisiti fondamentali che in prima approssimazione si possono cosí delineare:

a) gli obiettivi e il programma dei corsi dovranno essere definiti e concordati bilateralmente;

b) lo svolgimento dei corsi deve avvenire, in misura prevalente, in forma seminariale e ciò sia perché possano manifestarsi primariamente le esigenze e le proposte dei corsisti, sia perché si creano, cosí, abbondanti occasioni di sviluppo attivo della loro competenza linguistica;

c) per quanto riguarda la formazione di base degli insegnanti, la materia dei corsi deve colmare le eventuali lacune dovute a carenze del curricolo di formazione, carenze che di solito riguardano (vedi sopra 2.1.) non lo studio della lingua vera e propria e della letteratura, ma piuttosto il settore delle discipline linguistiche e la conoscenza della cultura e della società italiana sotto profili non specificamente letterari;

d) anche la preparazione psico-pedagogica e nelle metodologie didattiche, che gli insegnanti di italiano hanno scarsa possibilità di acquisire nei luoghi della loro formazione (vedi sopra 2.2.), dev'essere uno dei momenti qualificanti dei corsi di questo terzo livello;

e) in sede di aggiornamento devono essere affrontati temi specifici, di lingua e di cultura, utilizzabili direttamente nella pratica di insegnamento dei corsisti, ciò che richiede, appunto, una programmazione preliminare bilaterale dei corsi: le attività di studio, i dibattiti, le esperienze dirette a contatto con l'ambiente italiano, le gite culturali, ecc., devono poter fornire agli insegnanti una quantità di "materiali" da introdurre poi come contenuti più attuali e rispondenti alle situazioni delle proprie classi di insegnamento;

f) i corsi devono essere organizzati d'intesa, oltre che con i nostri Istituti di cultura, con i Dipartimenti di Italiano delle Università estere e cio per svariati motivi: perché presso tali istituzioni si trovano già, nei singoli paesi, gli operatori più qualificati nel campo dell'italianistica; perché gli insegnanti hanno cosí possibilità di restare, anche successivamente, in contatto con gli studi superiori del loro paese; perché in tal modo anche i Dipartimenti di Italiano vengono coinvolti nelle attività di aggiornamento degli insegnanti e nei problemi della didattica corrente dell'italiano. 
3. Altre prospettive per l'insegnamento dell'italiano.

E' constatazione fin troppo facile che l'italiano nelle Università estere $^{13}$ si insegna quasi esclusivamente per coloro che aspirano a diventare insegnanti di italiano, e in pochi casi, per coloro che si indirizzano agli studi di letterature e antichità classiche e di letterature romanze. Sono molto rari, infatti, i corsi di lingua italiana per studenti di altri Dipartimenti,

Un allargamento dell'area di studio dell'italiano si potrà ottenere lavorando in due direzioni, una linguistica e l'altra culturale, e cioè: da una parte moltiplicando e rafforzando i corsi di lingua italiana per studenti di altri Dipartimenti, ${ }^{14}$ da un'altra parte favorendo l'istituzione di corsi, non lingua italiana, di materie storiche, urbanistiche, economiche, politiche, incentrati sulla realtà italiana, in modo da richiamare su di essa l'attenzione di studenti di ben diverso orientamento. ${ }^{15}$ Le due direzioni, com'è ovvio, risulteranno prima o poi convergenti: un urbanista straniero interessato ai problemi dei nostri centri storici, o un economista che studi le caratteristiche delle medio-piccole imprese, cosí tipiche del nostro paese e cosí presenti nel nostro commercio estero, o un esperto in scienze politiche che si interessi al quadro politico europeo-mediterraneo dei nostri tempi, non potranno, dopo un po', trascurare lo studio dell'italiano.

\section{Conclusione.}

$\mathrm{Al}$ centro del nostro discorso è un tema ben preciso: la formazione degli insegnanti di italiano all'estero. Non è un tema di piccole dimensioni e non è certo separabile da altre questioni delicate e importanti, come la sicurezza di impiego per tali insegnanti (ne abbiamo, infatti, dovuto parlare) e la disponibilità di materiale didattico aggiorna to e valido. Ma il circolo chiuso delle varie difficoltà che gravano sul rendimento dei corsi di italiano va spezzato in qualche punto: e il punto di minor resistenza sembra la qualificazione degli insegnanti. In questa direzione possono essere concentrati utilmente, con risultati a più breve termine, gli sforzi sia da parte italiana sia da parte degli esponenti della cultura italiana all'estero.

Occorrono, naturalmente, piani per condurre questa operazione verso gli obiettivi desiderati. Con le considerazioni svolte nelle pagine precedenti si è proposto di dare alla formazione e qualificazione degli insegnanti un'impostazione che rispondesse chiaramente ai principi:

- del moderno concet to di "cultura", la quale, antropologicamente intesa, ricompone in uno stesso quadro fatti solitamente scissi e contrapposti nella nostra coscienza - come la cultura intellet- 
tuale e le manifestazioni della vita pratica, o le tradizioni culturali "egemoni" e quelle "subalterne" - fatti che vanno invece accostati, perché se ne possano cogliere i nessi, a volte dialettici, ma innegabili e fecondi;

- della psico-pedagogia e della glottodidattica più aggiornate e affinate che propugnano "la necessità di un supporto pluridisciplinare e interdisciplinare" per i processi di apprendimento delle lingue, processi da attuare tenendo pienamente conto di "tutti gli aspetti e fattori attivamente presenti nella situazione di apprendimento", cioè nel singolo sogget to che apprende e nella sua situazione; 16

- delle politiche di multiculturalismo in via di affermazione in molti paesi, politiche che hanno come principio ispiratore non una semplice disponibilità alla penetrazione culturale di altri paesi, ma l'intenzione di assicurare una più definita identità etnica a individui e gruppi che, dopo il grande strappo della migrazione, vivono nell'anonimo e immenso spazio delle società industrializzate. ${ }^{17}$ Una prospettiva, questa, che deve indurci a cambiare alcuni connotati alla nostra azione di "diffusione della lingua e cultura italiana", perché ci obbliga a tener conto delle attese e della dimensione culturale propria dei destinatari di quella azione.

\section{Università di Roma}

\section{NOTE}

* Testo della relazione conclusiva dei lavori del Convegno per l'organizzazione degli studi d'italiano in Canada, promosso dal Ministero degli Affari Esteri d'Italia, Toronto 17-21 Settembre 1979.

1 L'espressione è ripresa dall'intervento del Ministro Sergio Romano nel Convegno Immagine culturale dell'Italia all'estero, organizzato dal Ministero degli Affari Esteri e dalla Commissione Nazionale Italiana per I'Unesco, Roma 26 febbraio 1979; Atti, a c. di A. Bartole, (Roma), p. 115.

2 Preciso che le mie considerazioni riguardano i veri e propri insegnanti con titolo di studio universitario e non tutti coloro che semplicemente "insegnano italiano" nelle scuole. $\grave{E}$ noto infatti che da quando sono stati istituiti i corsi integrativi per l'attuazione dei nuovi programmi di multiculturalismo (vedi 1.3, punto b), per far fronte all'improvvisa richiesta di molti insegnanti, i corsi di italiano nelle scuole sono stati affidati in molti casi a persone prive di un regolare titolo di studio. Queste situazioni anomale (ma diffuse!) non rientrano nel quadro che qui si prospetta.

$3 \mathrm{Cfr}$. le relazioni dei professori: E. Musacchio per l'Alberta, S. De Stefanis Ciccone per la British Colombia, M. Danesi per l'Ontario, G. Villani per il Québec. La situazione è stata descritta negli stessi termini, in altre occasioni, per vari altri paesi (USA, Inghilterra, Germania). 
4 Quasi sempre l'"italofonia" di questi soggetti consiste nell'uso di un dialetto familiare abbastanza impoverito e con le variazioni (soprattutto lessicali e semantiche) subite a contatto con la lingua dominante del paese ospitante: in pratica, l'italiano parlato dagli oriundi è il cosiddetto italiese. - Sulla condizione di "semibilinguismo," propria dei bambini e ragazzi dei nostri emigrati (sia pure vista in contesto europeo), cfr. A. Tosi. "Bilinguismo e immigrazione: una nota sociolinguistica al piano europeo di mantenimento delle lingue nazionali nelle comunità di emigrati," in Rassegna italiana di linguistica applicata, XI-XII (19791980), 243-63.

5 E stato notato che tale confronto è spesso svantaggioso proprio per gli oriundi italiani. Ė bene chiarire che il rendimento migliore, o almeno più rapido, degli alunni non oriundi italiani dipende (oltre che, talora, da un migliore ambientamento scolastico generale) semplicemente dal fat to che l'insegnamento dell'italiano è svolto con metodi e con testi rivolti proprio a soggetti di lingua materna straniera. In altri termini, i metodi e i testi si adattano perfettamente allo studente di lingua materna inglese (o francese, o spagnola) e ignorano le interferenze create dai nostri dialetti. Ma si verifica anche il caso inverso: di non oriundi che si sentono svantaggiati rispetto agli oriundi, che sono più familiarizzati col sistema linguistico italiano e con la cultura italiana. - Gli esperti di queste situazioni sostengono che sia nettamente preferibile dividere gli oriundi (impropriamente detti "italofoni") dai non oriundi, almeno ai livelli iniziali, per ricongiungerli ai livelli più avanzati.

6 Viene segnalato dappertutto una forte tendenza all'abbandono dello studio dell'italiano dopo i primi periodi di frequenza ai corsi. Il fenomenso si verifica anche, e marcatamente, all'Università.

7 Con "atteggiamento" va reso il termine inglese attitude in uso nella sociologia e psico-pedagogia anglosassone (ma il termine è stato attinto dal francese) per indicare un sistema di "reazioni di valutazione d'ordine affettivo" basato sui concetti e le credenze relativi a un dato oggetto, come puo essere un gruppo sociale con i suoi costumi e la sua lingua. Cfr. M.E. Shaw e J.M. Wright, Scales for the Mensurement of Attitudes (New York, 1967), p. 3. Ai problemi di "atteggiamento e motivazione" (di bambini e adolescenti) nell'apprendimento di una seconda lingua in situazioni di convivenza con altri gruppi etnici è dedicato un intero numero di The Canadian Modern Language Review/La revue canadienne des langues vivantes, vol. 32, num. 3, (febbr. 1976).

8 Ho anticipato alcune osservazioni in proposito nel mio intervento al Convegno Immagine culturale dell'Italia all'estero; cfr. il cit. vol. di Atti, pp. 149-51. Altre considerazioni, in precedenza, in F. Sabatini, LM. Lombardi Satriani, R. Simone, "Emigrazione italiana, lingua e processi di acculturazione in Europa (Considerazione sullo status internazionale dell'italiano)," nel vol. Italiano d'oggi. Lingua non letteraria e lingue speciali. Centro per lo studio dell'insegnamento all'estero dell'italiano (Università di Trieste, Trieste 1974), pp. 111-32; F. Sabatini, "Per l'educazione linguistica della seconda generazione degli emigrati," in AA. VV., La lingua degli emigrati, a cura di L. Zanier (Firenze, 1977).

9 Per i problemi di recupero di una identità etnica at traverso l'Heritage Language Program cfr. il vol. di Berry, Kalin e Taylor citato nella nota 17.

10 Alcune sono segnalate nella succesiva nota 12 .

$11 \mathrm{Per}$ un rapido profilo delle conseguenze di questo processo cfr. F. Sabatini, "Linee di tendenza dell'italiano cntemporaneo e problemi di norma," nel vol. La lingua italiana in Finlandia, Atti del primo convegno degli insegnanti di italiano in Finlandia, Turku 17 e 18 maggio 1979, (Turku, 1980), pp. 73-91.

12 Sono da segnalare le seguenti iniziative: Nell'University of Toronto sono impartiti, nel Dipartimento di Italiano, anche corsi di Italian Linguistics; History of the Italian Language; Italian Cinema; Italian Culture and Civilization (anche in corsi estivi tenuti a Siena) e, in altri Dipartimenti, corsi su: Modern Italy: The Italocanadians. Nella York University di Toronto il corso di Modern Italian Literature and Culture tratta sostanzialemente temi della realtà politico-sociale.

Nella McGill University di Montréal, nel Dipartimento di Italiano sono 
impartiti corsi di Storia della lingua italiana e su Cinema e società nell'Italia di oggi. Nell'Université de Montréal sono stati introdotti nel 1979 un corso di Dialettologia italiana (nel Dipartimento di Linguistica) e un corso di Storia dell'Italia contemporanen (nel Dipartimento di Storia).

Nell Concordia University di Montréal, nel Dipartimento di Italiano si tiene un corso su Some aspects of contemporary Italian life.

13 Dal panorama di questa relazione sono esclusi tutti gli altri tipi di corsi che si tengono a cura degli Istituti di cultura, della "Dante Alighieri" e di altre Istituzioni. I destinatari e le finalità di questi corsi sono di vario tipo, ma certamente esistono connessioni anche con il mondo universitario e della scuola.

14 I corsi elementari di lingua italiana sarebbero, di per sé, adatti anche a studenti di altri Dipartimenti e sono infatti aperti ad essi; vi sono, raramente, anche veri cours de service di italiano (ad es. nell'Université de Montréal). Ma si dovrebbe in ogni caso introdurre una caratterizzazione particolare di questi corsi in funzione dall'utilizzazione che gli studenti di altri Dipartimenti intendono fare dell'italiano: ad es., con lettura di libri o presentazione di filmati su argomenti di loro interesse.

15 E un buon segno che nell'University of Toronto (sia nella sede centrale che nello Scarborough College) si tengano già corsi, in lingua inglese, sulla letteratura italiana medievale e rinascimentale, sul teatro e sul cinema italiano e su un tema come Modern Italy. Su questa scia si dovrebbero prendere iniziative per corsi di materie di altro genere. Infatti, che in altri settori di studi (architettura, design, scienze politiche ed economiche, sociologia, storiografia, archeologia) e in taluni campi di attività (turismo, esportazione di prodotti artigianali, ecc.) vi sia da tempo un particolare "interesse per il fatto italiano," come ha detto Italo Calvino, è una circostanza ben nota: ma a fronte di questo fenomeno mancano iniziative che aiutino la diffusione della nostra lingua. Questa situazione è stata rilevata più volte: ad es. da Sergio Romano, nel discorso" La lingua e la cultura italiana nel mondo d'oggi" (pronunciato a Roma il 12 ottobre 1978, sotto gli auspici del Centro di Studi per la Conciliazione Internazionale; testo pubbl. in opuscolo a c. del Banco di Roma, s.d.) e da vari relatori e intervenuti al cit. Convegno sull'Immagine culturale dell'Italia all'estero.

16 La linea dell'"approccio integrato," proposta decisamente già negli anni Sessanta, risulta oggi piu chiaramente vincente: vedi la sintesi critica di $R$. Titone, Glottodidattica. Un profilo storico, (Minerva Italica, Bergamo, 1980: a p. 188 le espressioni da me citate nel testo).

17 E il concetto della "new ethnicity," discusso recentemente (dopo il lancio della politica di multiculturalismo nel 1971) soprattutto da vari autori canadesi. Cfr. per tutto il problema J.W. Berry, R. Kalin e D.M. Taylor, Multiculturalism and Ethnic Attitudes in Canada (Ottawa, 1976), specialmente alle pp. 11-15, 131 ss.e 248. 